\title{
Framing Effects Influence Interface Feature Decisions
}

\author{
Andy Cockburn \\ University of Canterbury \\ Christchurch, New Zealand \\ andy@cosc.canterbury.ac.nz
}

\author{
Blaine Lewis \\ University of Toronto \\ Toronto, Canada \\ blaine@dgp.toronto.edu
}

\author{
Philip Quinn \\ Google \\ Mountain View, CA \\ philip@quinn.gen.nz
}

\author{
Carl Gutwin \\ University of \\ Saskatchewan \\ Saskatoon, Canada \\ gutwin@cs.usask.ca
}

\begin{abstract}
Studies in psychology have shown that framing effects, where the positive or negative attributes of logically equivalent choices are emphasised, influence people's decisions. When outcomes are uncertain, framing effects also induce patterns of choice reversal, where decisions tend to be risk averse when gains are emphasised and risk seeking when losses are emphasised. Studies of these effects typically use potent framing stimuli, such as the mortality of people suffering from diseases or personal financial standing. We examine whether these effects arise in users' decisions about interface features, which typically have less visceral consequences, using a crowd-sourced study based on snap-to-grid drag-anddrop tasks $(n=842)$. The study examined several framing conditions: those similar to prior psychological research, and those similar to typical interaction choices (enabling/disabling features). Results indicate that attribute framing strongly influences users' decisions, that these decisions conform to patterns of risk seeking for losses, and that patterns of choice reversal occur.
\end{abstract}

\section{Author Keywords}

Framing effects; interface decisions; attribute framing; risky choice framing.

\section{CCS Concepts}

- Human-centered computing $\rightarrow$ Human computer interaction (HCI); HCI theory, concepts and models; Empirical studies in HCI.

\section{INTRODUCTION}

To help users become more efficient in their tasks, interfaces often include decision mechanisms that allow users to enable or disable features that offer some form of automation or assistance. For example, Figure 1 shows options in Microsoft Word that allow users to choose whether or not bulleted and numbered lists are automatically inserted when the system detects that a user may be trying to create them. A user's decision to enable or disable such features can have important implications for their future efficiency with the interface.

Prior research from behavioural economics and psychology has shown that human decisions reliably deviate from what might be considered objective or 'rational' behaviour due to

Permission to make digital or hard copies of part or all of this work for personal or classroom use is granted without fee provided that copies are not made or distributed for profit or commercial advantage and that copies bear this notice and the full citation on the first page. Copyrights for third-party components of this work must be honored. For all other uses, contact the owner/author(s). Copyright is held by the author/owner(s).

CHI'20, April 25-30, 2020, Honolulu, HI, USA.

(C) 2020 Copyright is held by the owner/author(s).

ACM ISBN 978-1-4503-6708-0/20/04..

DOI: http://dx.doi.org/10.1145/3313831.3376496

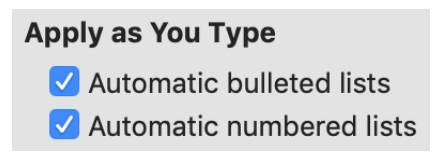

Figure 1. A preference control supporting user decisions about interface features in Microsoft Word.

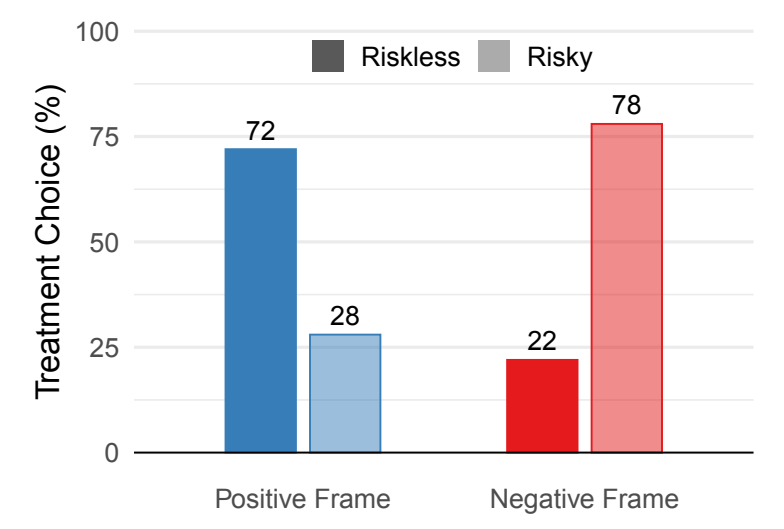

Figure 2. Tversky and Kahneman's framing results: risk aversion with positive frames, risk seeking with negative frames.

framing effects. Figure 2 summarises results from Tversky and Kahneman's now-famous study of subjects' decisions between certain (riskless) and uncertain (risky) choices that were framed positively or negatively [31]. When faced with a fictitious scenario in which an infectious disease was expected to kill 600 people, a majority of subjects chose a treatment programme in which '200 people will be saved' over one in which 'there is $1 / 3$ probability that 600 people will be saved, and $2 / 3$ probability that no people will be saved' - that is, in the context of gains (saving lives), subjects were averse to the risky programme despite the same expected outcome. However, in the domain of losses these preferences were reversed: a minority of subjects chose a programme in which ' 400 people will die' over one in which 'there is $1 / 3$ probability that nobody will die, and $2 / 3$ probability that 600 people will die'.

Similar findings have been replicated in various experimental settings [18], with Tversky and Kahneman referring to a 'pattern of risk aversion in choices involving gains and risk seeking in choices involving losses' [31, p. 454]. People prefer a certainty in smaller gains to a gamble on larger gains with the risk of no gain (risk aversion); and conversely, people prefer a gamble on the possibility of no loss with the risk of larger losses to a certainty in a smaller loss (risk seeking). 


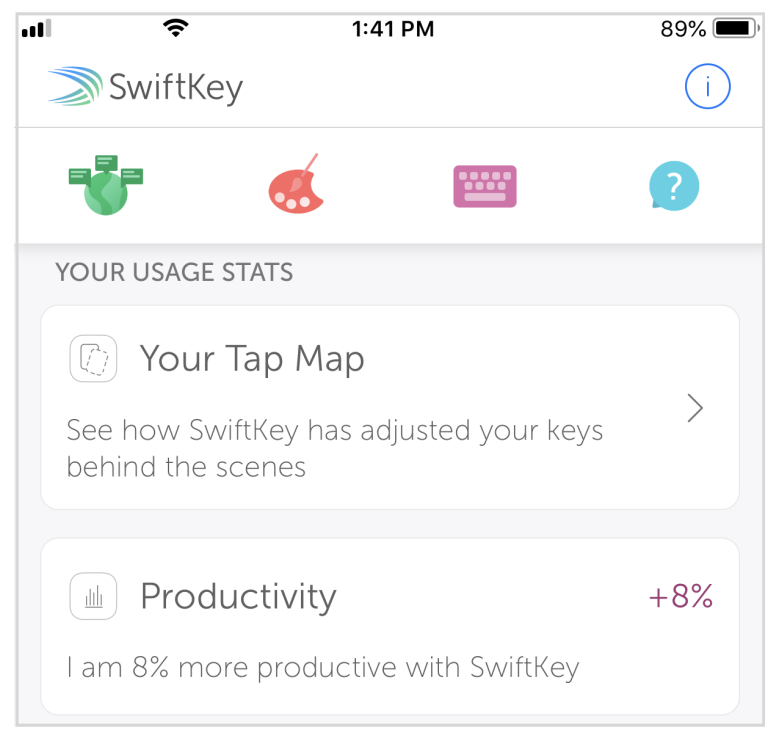

Figure 3. Framed productivity statistics on the startup page of the SwiftKey mobile keyboard (swiftkey. com).

These findings suggest that users' decisions to enable or disable user-interface features may be similarly influenced by these two factors: framing and risk. Framing concerns the way in which a choice is presented to a user, with positive framing emphasising the gains associated with using an interface feature (e.g. 'you will be $8 \%$ more productive with this feature'; see Figure 3) and negative framing emphasising losses (e.g. 'you will be $8 \%$ less productive without this feature'). If the feature will be helpful in a majority of future tasks then it makes sense for the user to enable it; but otherwise not.

Risk concerns the degree to which the outcome of a decision is knowable in advance: an interface choice is riskless if a user is certain that a feature will help in future tasks (or certain that it will hinder), otherwise the choice is risky. Although this use of the term 'risk' for uncertain outcomes is unusual in human-computer interaction, we adopt it for consistency with the prior psychological literature. Making a choice to enable or disable an interface feature often requires a user to speculate about their future interactions, the reliability of the system, and the net gains/losses to their productivity. In some cases a user will know what their future tasks will entail, how the system will support them, and how much they will gain or lose from a feature - and therefore the choice is riskless; but in many cases upcoming tasks are uncertain and the system's behaviour is unpredictable - in which case the choice is risky.

Although previous psychological studies have shown that framing and risk have well-demonstrated effects on human decisions (reviewed below), there are reasons to suspect that the findings may not generalise to interaction. First, the experimental methods used in these studies have typically used strong stimuli, such as choices involving life and death, or financial gains and losses. In contrast, user interaction is typically less visceral and the potency or existence of framing effects is unclear if the consequences of a decision are relatively minor (e.g. a small inconvenience, frustration, or loss of time). Second, psychological studies typically examine decisions between two directly opposing frames, whereas interaction decisions will often present a single frame and ask users to decide whether to accept or reject a feature. Understanding the potential effects of framing and risk on user interface decisions is important for interface designers. With access to this knowledge, designers have an additional tool to assist users in making good decisions regarding the features they enable.

We conducted a crowd-sourced experiment $(n=842)$ to investigate the influence of framing and risk on interaction choices. The experiment used interaction with snap-to-grid functionality in drag-and-drop tasks, and used framing conditions that closely aligned with Tversky and Kahneman's original method (but in an interactive setting) and framing conditions that were ecologically consistent with how choices to enable/disable features are presented in user interfaces.

Results supported the hypotheses, leading to the following specific contributions. First, we review psychology studies of framing effects and explain why the findings may be relevant to users' decisions to accept or reject interface features. Second, we experimentally demonstrate that (1) positive and negative attribute framing influences decisions during interaction, (2) users have a tendency for risk seeking with negative frames, and (3) this tendency contrasts with their decisions under positive frames. Third, we discuss implications for design based on the findings.

\section{BACKGROUND}

\section{Psychological Foundations}

In 1973, Harris [7] observed that the way in which quantitative questions were framed influenced subjects' mean responses. For example, when asked to estimate 'how high was [a] plane flying', the mean response was $8,907 \mathrm{ft}$; but when 'high' was changed to 'low', the mean response dropped to 4,481 ft. Related effects were soon replicated by Loftus [20], who demonstrated the ease with which eyewitness reports of accidents or classroom disruptions could be influenced.

Tversky and Kahneman's [31] studies (summarised in the Introduction) advanced the understanding of framing effects in two key ways. First, they demonstrated choice reversal for positive and negative frames in the presence of risk - subjects' responses were characterised by risk aversion for positive frames and by risk seeking for negative frames. Second, they analysed these results within their prospect theory [13, 32], which explains how people evaluate choices concerning prospective gains and losses.

With increasing experimental replication and methodological variation across studies, Levin et al. [18] developed a typology of these framing effects: attribute framing, risky-choice framing, and goal framing.

Attribute framing is the simplest form of framing in which positive or negative attributes of an outcome are emphasised in their description. For example, describing beef as ' $75 \%$ lean' versus ' $25 \%$ fat' [17]. Attribute framing effects are weak when directed at a person's strongly held views, such as rights to abortion [21], or when directed at their own performance 
(e.g. 'percentage right' vs. 'percentage wrong' [16] or cheating in exams [19]). This latter point raises questions regarding the potency of framing effects when users make decisions about their own performance with interactive systems.

Risky-choice framing is the type exemplified by Tversky and Kahneman's aforementioned study [31]. Risky-choice framing includes the occurrence of choice reversal in which people are risk averse for gains (preferring certain gains to the risk of possibly larger gains or nothing at all), but risk seeking for losses (preferring the possibility of larger losses with the chance of no loss to the certainty of a small loss).

Goal framing concerns persuasiveness towards a particular behaviour, and while it is likely to be relevant for persuasive and behaviour-change interfaces, it is not directly relevant to our present studies (see Levin et al. [18] for further details).

\section{Applications in Human-Computer Interaction}

Several studies in human-computer interaction have directly or tangentially examined framing effects. Jameson et al. [12] provide a comprehensive review and framework for understanding user decisions during interaction, including a brief discussion of framing effects. Acknowledging that designers may unintentionally influence decisions through framing, they note 'When the goal is not persuasion but choice support, the idea is to avoid introducing unnecessary distortion via framing effects - even though it is in general difficult or impossible to find a completely neutral framing of any consequence' [12, p. 90].

A significant portion of prior work on framing in humancomputer interaction concerns goal framing and its role in persuasive design and behaviour-change interfaces. Nyström and Mustaquim [24] provide an introduction to the application of framing for persuasive design, with a focus on sustainability practices; however, their model is not supported by independent empirical evidence. Choe et al. [3] also investigated persuasive design, examining how framing effects can be used to 'nudge' users away from privacy-invasive mobile applications. Their interventions focus on the use of brief iconic visualisations (e.g. thumbs-up and thumbs-down symbols), with evaluation results confirming successful influence on people's decisions. Other persuasive systems making explicit use of framing have been evaluated in domains including personal productivity [15] and nutritional choices [11].

Some attribute framing studies have also demonstrated that user assessments are influenced by framing, including product assessments (e.g. [27]) and user judgements regarding website quality and interface aesthetics [5, 8-10].

However, although framing effects are empirically well established in psychological research, their generalisation to human-computer interaction is challenged by three key differences in experimental practice between the two fields.

First, psychological studies have typically used powerful emotional stimuli, such as mortality, financial risk, and personal reputation. In contrast, decisions made during interaction are seldom so visceral - instead, users typically make decisions that have relatively minor implications relating to frustration and efficiency.
Second, psychological studies typically examine a direct choice between two opposing frames, such choosing between ' 400 people will die' and ' $2 / 3$ probability that 600 people will die... '. However, during interaction there is likely to be only a single frame, with the choice being to accept or reject a feature. For example, users may be asked to choose between enable feature and disable feature in response to the frame: 'you will be $8 \%$ faster when using this feature'. Their decision may be different if the frame were instead 'you will be $8 \%$ slower without this feature'.

Third, subjects in psychological studies rarely experience the outcomes of their decisions - the choice between '200 people will be saved' and ' 400 people will die' has no factual consequence. However, in interaction experiments it is easy to expose subjects to experimental conditions that represent the outcome of their decision.

\section{FRAMING EXPERIMENT}

We conducted an experiment to examine whether framing effects influence users' decisions related to interface features. The experiment examined both attribute framing and riskychoice framing to understand how decisions around interface features are influenced when choices are framed positively or negatively, and by outcome uncertainty. In our experiment, riskless choices involved certainty about the content of upcoming tasks, whereas risky choices involved uncertainty.

Subjects initially completed two sets of drag-and-drop tasks, one with a snap-to-grid feature turned on and another with the feature off (described below). They then made a choice between two options regarding their preference for a further set of drag-and-drop tasks. The experiment covered a variety of different framing conditions for the choice, as summarised in Table 1 and further described below.

\section{Hypotheses}

The hypotheses tested were as follows:

$H_{1}$ Attribute Framing: Under a riskless choice, positive frames (emphasising gains) will lead to a higher acceptance than negative frames.

$\mathrm{H}_{2}$ Risk Aversion for Gains: Riskless positive frames will result in a higher acceptance than risky positive frames. That is, when positive attributes are emphasised (gains), subjects will prefer to capitalise on certain gains rather than to risk them for potentially larger gains.

$\mathrm{H}_{3}$ Risk Seeking for Losses: Risky negative frames will result in a higher acceptance than riskless negative frames. That is, when negative attributes are emphasised (losses), subjects will prefer to risk potentially larger losses for the chance of avoiding certain losses.

$\mathrm{H}_{4}$ Choice Reversal: Willingness to accept risk will reverse depending on whether the frame is positive or negative, consistent with risk aversion for positive frames $\left(\mathrm{H}_{2}\right)$ and risk seeking for negative frames $\left(\mathrm{H}_{3}\right)$.

These hypotheses reflect the patterns shown in Figure 2.

\section{Task Interactions}

We based our experimental method on object drag-and-drop interactions, with and without a snap-to-grid feature. We used drag-and-drop and snap-to-grid interactions because 
they are simple and familiar interactions, their performance and mechanical requirements are easily controlled, and they have been used in previous experiments investigating interface preferences [26]. Similar drag-and-drop and snap-to-grid features can be found in most interfaces that support drawing and manipulating graphical objects (e.g. Microsoft PowerPoint).

Experimental tasks involved dragging a $50 \times 50 \mathrm{px}$ filled black square to a target location indicated by a second $50 \times 50 \mathrm{px}$ hollow square (Figure 4). The distance between the initial location of the object and the target was $256 \mathrm{px}$, but the direction was randomised. The black square turned blue while it was being dragged, and turned green when its location exactly matched that of the target.

There were two main variants of drag-and-drop tasks: neutral and snapping. During neutral tasks, the object appeared to move smoothly and continuously when dragged, ${ }^{1}$ and during snapping tasks the object moved in discrete units when dragged - snapping between locations to maintain alignment with an invisible grid that was $60 \mathrm{px}$ wide.

There were also two variants of snapping tasks: aligned and misaligned. In aligned tasks the target location was congruent with the underlying $60 \mathrm{px}$ grid. These tasks represent successful use of the snapping feature, in which user performance is faster and less effortful than in neutral tasks because the dragged object snaps onto the target when dragged to its vicinity. In misaligned tasks the target location was offset from the grid. Completing these tasks required that the snapping be disabled, which was achieved by dropping the object (i.e. releasing the mouse button or lifting a finger from a trackpad), and then pressing and holding the 'Shift' key on the keyboard while re-selecting the object. This enabled neutral behaviour, allowing the user to complete the task by smoothly dragging the object to the target. Completing misaligned snapping tasks was intentionally slower and more effortful than neutral tasks.

All tasks (including misaligned tasks) had to be initiated without the 'Shift' key pressed. Subjects only learned that a task was misaligned after observing that the dragged object would not align with the target.

\section{Procedure}

The experiment was administered on Amazon Mechanical Turk through the subject's web browser. Amazon Mechanical Turk is a system that acts as a broker between requesters and remote workers who complete paid human intelligence tasks.

The main elements of the procedure are summarised in Figure 5. Each subject received a brief introduction to the experiment, informing them that it concerned preferences for drag-and-drop interactions with and without snap-to-grid assistance. They then responded to questions regarding their age, sex, and pointing device before proceeding to a training tutorial. The tutorial described how to complete drag-anddrop tasks with each of the three main conditions under study - neutral, snapping-aligned, and snapping-misaligned. Subjects could only advance after successfully completing three

\footnotetext{
${ }^{1}$ The object actually moved at a 4 px resolution to avoid imposing the unnec-
} essarily high accuracy requirements that would occur at a 1 px resolution.

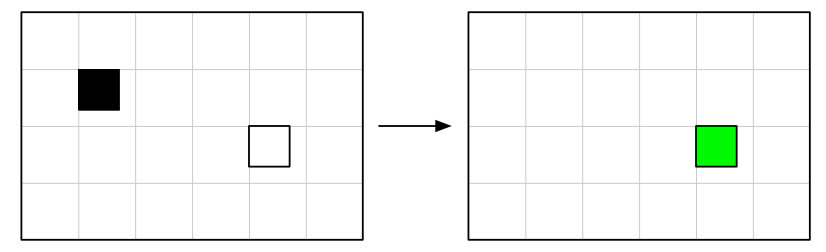

(a) Aligned snapping: the target was aligned with a grid that the object snaps to when dragged.

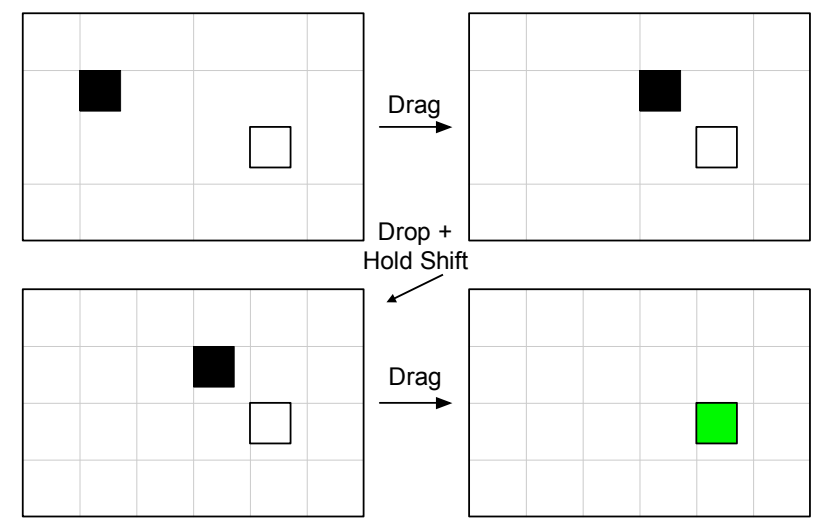

(b) Misaligned snapping: the target was misaligned with the grid, preventing direct placement. Dropping and reacquiring with the 'Shift' key held down reduced the size of the grid.

Figure 4. Drag-and-drop tasks involved dragging a filled black square onto a hollow target square. The grid, shown here for conceptual clarity, was not visible to subjects.

drag-and-drop tasks with each of these conditions, including the requirement to drop the dragged object and reacquire it with the 'Shift' key held down (snapping-misaligned). After the practice tasks, the main conditions began (steps 2 and 3 in Figure 5). The presentation order of 12 neutral and 12 snapping tasks was counterbalanced (odd numbered subjects did neutral tasks first; even snapping first).

Within each set of 12 tasks, successfully completing one task (by aligning the dragged object with the target and dropping it) automatically initiated the next. The 12 snapping tasks contained four aligned tasks and eight misaligned tasks. We used an umbalanced number of aligned and misaligned tasks because results from prior and preliminary studies (see the Discussion) showed a positivity bias towards snapping - preferring it even when it impaired performance [26]. We therefore intended that the 4-to- 8 split of aligned and misaligned tasks would avoid ceiling or floor effects in subjects' acceptance of the snapping behaviour.

After completing the second set of tasks, the main framing question was presented (step 4 in Figure 5). To isolate the existence and influence of framing effects during interaction, the framing conditions covered a spectrum of relatively subtle changes in their presentation, as summarised in Table 1.

In the 'Tversky \& Kahneman' frames (rows 1 and 2 of Table 1), subjects chose between two opposing frames that represent different ways of expressing outcomes that are logically equivalent. These frames were included to determine whether framing effects occur when users make decisions around the 


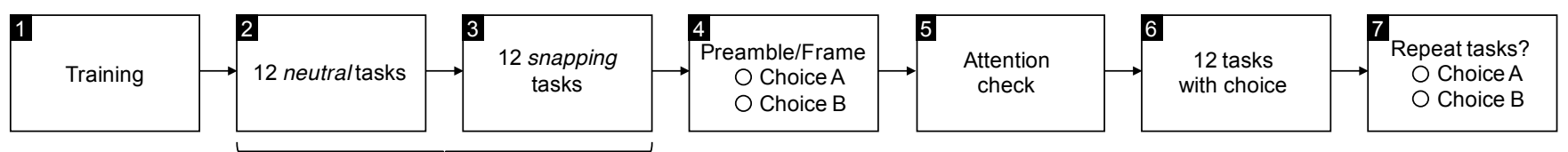

Counterbalanced

Figure 5. Procedure for the experiment: (1) subjects received a description of the task and interface behaviour and completed (2) a block of 12 neutral tasks counterbalanced with (3) a block of 12 snapping tasks. They were then (4) asked to select between one of the choices described in Table 1. After (5) responding to an attention check question regarding the number of upcoming tasks, and then completed (6) a block of 12 tasks under their decision. Finally, (7) subjects were asked if they would change their decision if repeating the tasks once more.

Tversky \& Kahneman Framing

\begin{tabular}{|c|c|c|c|c|c|c|c|}
\hline & Type & $n$ & Preamble & Choice A & \multicolumn{3}{|l|}{ Choice B } \\
\hline 1 & Attribute & $\begin{array}{l}50 \\
(136)\end{array}$ & \multirow{3}{*}{$\begin{array}{l}\text { You are about to carry out } \\
\text { a new set of } 12 \text { tasks. } \\
\text { Grid snapping will be on. } \\
\text { Would you prefer? }\end{array}$} & $\begin{array}{l}\text { [Positive }] \text { The tasks will include } 4 \text { aligned } \\
\text { with the grid. }\end{array}$ & \multicolumn{3}{|c|}{$\begin{array}{l}\text { [Negative] The tasks will include } 8 \text { misaligned } \\
\text { with the grid. }\end{array}$} \\
\hline $\begin{array}{l}2 \\
\mathrm{a}\end{array}$ & $\begin{array}{l}\text { Risky Choice } \\
\text { Positive }\end{array}$ & $\begin{array}{l}\mathrm{Y} \\
\mathrm{a} \\
\mathrm{C} \\
\mathrm{V}\end{array}$ & & $\begin{array}{l}\text { [Riskless] The tasks will include } 4 \text { aligned } \\
\text { with the grid. }\end{array}$ & \multirow{2}{*}{\multicolumn{3}{|c|}{$\begin{array}{l}{[\text { Risky] There is a } 33 \% \text { chance that all tasks }} \\
\text { will be aligned with the grid, and a } 66 \% \text { chance } \\
\text { that no tasks will be aligned with the grid. } \\
\text { [Risky] There is a } 66 \% \text { chance that all tasks will } \\
\text { be misaligned with the grid, and a } 33 \% \text { chance } \\
\text { that no tasks will be misaligned with the grid. }\end{array}$}} \\
\hline $\mathrm{b}$ & Negative & 77 & & $\begin{array}{l}\text { [Riskless] The tasks will include } 8 \text { mis- } \\
\text { aligned with the grid. }\end{array}$ & & & \\
\hline \multicolumn{8}{|c|}{ UI-Style Framing } \\
\hline & Type & $n$ & Frame & & & Choice A & Choice B \\
\hline 3 & Attribute & & $\begin{array}{l}\text { You are about to carry } \\
\text { Would you like to turr }\end{array}$ & $\begin{array}{l}\text { out a new set of } 12 \text { tasks. } \\
\text { grid snapping on or off? }\end{array}$ & & & \\
\hline a & Baseline & 76 & The tasks will include & 4 aligned with the grid and 8 misaligned with & the grid. & Snapping On & Snapping Off \\
\hline $\mathrm{b}$ & Positive & $78(149)$ & The tasks will include & 4 aligned with the grid. & & Snapping On & Snapping Off \\
\hline $\mathrm{c}$ & Negative & $72(169)$ & The tasks will include & 8 misaligned with the grid. & & Snapping On & Snapping Off \\
\hline 4 & Risky Choice & & $\begin{array}{l}\text { You are about to carry } \\
\text { Would you like to turr }\end{array}$ & $\begin{array}{l}\text { out a new set of } 12 \text { tasks. } \\
\text { grid snapping on or off? }\end{array}$ & & & \\
\hline a & Positive & 76 & $\begin{array}{l}\text { There is a } \mathbf{3 3} \% \text { chanc } \\
\text { that no tasks will be }\end{array}$ & $\begin{array}{l}\text { that all tasks will be aligned with the grid, and } \\
\text { ligned with the grid. }\end{array}$ & a $66 \%$ chance & Snapping On & Snapping Off \\
\hline $\mathrm{b}$ & Negative & 82 & $\begin{array}{l}\text { There is a } 66 \% \text { chan } \\
\text { chance that no tasks }\end{array}$ & $\begin{array}{l}\text { e that all tasks will be misaligned with the gr } \\
\text { ill be misaligned with the grid. }\end{array}$ & id, and a $\mathbf{3 3 \%}$ & Snapping On & Snapping Off \\
\hline
\end{tabular}

Table 1. The experimental frames and choices, and the number of subjects analysed in each condition (with the total number in parentheses - see text).

relatively weak subjective experiences and outcomes of interaction. However, users seldom make decisions based on such an explicit and direct contrast between outcomes (i.e. users are unlikely to be asked to choose which tasks they want to complete). Instead, users typically make decisions to enable/disable features based on a presumption about future tasks. The experiment therefore also included 'UI-style' frames (rows 3 and 4) to provide insight into how framing effects may arise during ecologically valid interaction choices.

Having made their decision by selecting one of two radio buttons, an attention check question was presented (step 5), asking subjects to type the number of tasks that they will need to complete (the correct answer being ' 12 '). They then completed a final set of 12 tasks using their decision. Finally, after doing so, they responded to a question: 'If you were to repeat those tasks one more time, would you choose...', followed by the choice options previously shown.

\section{Subjects and Apparatus}

A total of 842 crowd-workers participated in the study. Their mean age was 36.0 years (SD 10.7); 290 (34\%) were female (8 declined to answer). In response to a question regarding their input device, $683(81 \%)$ reported using a mouse, $155(18 \%)$ a trackpad, and 4 some other device (e.g. trackball). The majority reported using Microsoft Windows (84\%), followed by Apple macOS (10\%), and Unix (5.5\%). Subjects completed the experiment in around 5 minutes, and were rewarded with a payment of USD $\$ 1.25$ (apportioned from an hourly rate of USD $\$ 15$ per hour).

Each subject was limited to at most one experimental condition (between-subjects design). Between-subjects treatment is strongly advised in experiments of this type to avoid crosscondition contamination [14].

The number of subjects completing tasks in each condition ranged from 75-169 (shown in Table 1). The framing state- 
ments used in conditions $1,3 b$, and $3 c$ were potentially sensitive to subject misreading the frames - for example, if choice A of condition 1 was misread as 'The tasks will be 4 aligned with the grid' instead of the actual frame 'The [12] tasks will include 4 aligned with the grid', then a subject should clearly prefer choice A to choice B (preferring 4 tasks to 8 ). We therefore excluded subjects who gave incorrect responses to the attention-check question and recruited additional subjects in these conditions to give a sample size of 50 in condition 1 and approximately 75 in conditions $3 \mathrm{~b}$ and $3 \mathrm{c}$ (shown in parentheses in Table 1). In the analysis of decisions reported below, we excluded the data from subjects who gave an incorrect response to the attention check question.

\section{RESULTS}

Results are analysed using traditional statistical methods, including analysis of variance (for measures of trial time) and $\chi^{2}$ tests for choice patterns. We report exact $p$ values derived from statistical tests, except when they are minuscule. However, we intentionally refrain from dichotomous interpretation of $p$ with respect to a predetermined $\alpha$ [33]. In adopting this approach, we intend to assist the reader in forming their own opinion about the strength of the results, although we do use phrases such as 'high confidence' to signify results where a $p$ value indicates that the observed data should seldom occur if the null hypothesis of no effect were true.

\section{Manipulation Check: Times}

The method assumes that snapping is beneficial (compared to neutral dragging) when targets are aligned with the grid, and that it is detrimental when they are misaligned. As a manipulation check, we examined neutral and snapping trial times when targets were aligned and misaligned with the grid (Figure 6). Neutral trial times were similar regardless of grid alignment, with means of $3.42 \mathrm{~s}(95 \% \mathrm{CI}[3.30,3.53])$ and $3.51 \mathrm{~s}(95 \%$ CI $[3.39,3.63])$ for aligned and misaligned tasks, respectively. As intended, snapping tasks were faster than neutral tasks when aligned with the grid (mean $2.69 \mathrm{~s}, 95 \% \mathrm{CI}$ $[2.44,2.95]$ ) and slower when misaligned (mean $6.01 \mathrm{~s}, 95 \%$ CI [5.72, 6.30]). On average, subjects were approximately $18 \mathrm{~s}$ slower across the 12 tasks with snapping than with neutral dragging (suggesting, perhaps, that subjects should objectively

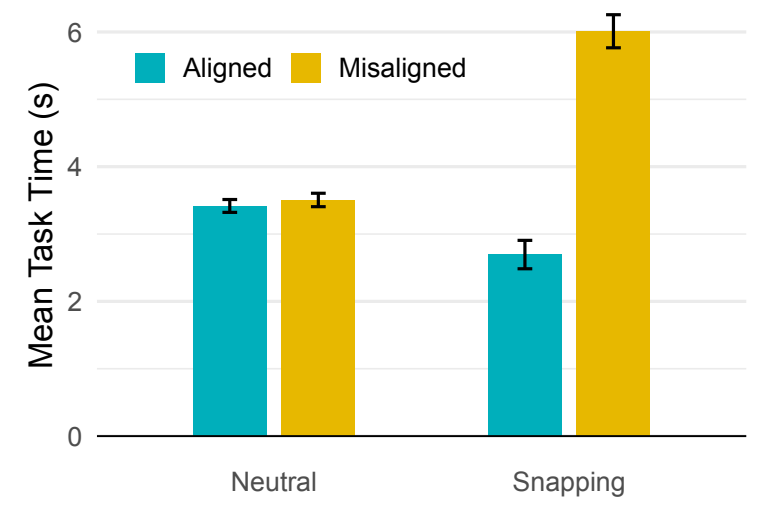

Figure 6. The mean times for neutral and snapping tasks when targets were aligned or misaligned with the grid $( \pm 95 \% \mathrm{CI})$. choose to turn snapping off [cf. 26]). A $2 \times 2$ ANOVA of the interaction between technique $\in\{$ snapping, neutral $\}$ and trial-type $\in\{$ aligned, misaligned $\}$ shows $F_{1,841}=319.0$, $p<1 \times 10^{-5}$.

\section{Tversky \& Kahneman Attribute Framing}

Tversky \& Kahneman attribute framing (row 1 of Table 1) required subjects to choose between a positive frame that emphasised tasks in which snapping was beneficial (" 4 [tasks] aligned with the grid') and a negative frame that emphasised tasks in which snapping was harmful (' 8 [tasks] misaligned with the grid'). The preamble to the frame stated that they would be completing 12 tasks and that snapping would be on, so both choices describe logically equivalent outcomes for the user. A majority (84\%) chose the positive frame in preference to the negative frame (Figure 7a), supporting $H_{1}$ : $\chi^{2}(1)=21.8, p<1 \times 10^{-5}$.

\section{Tversky \& Kahneman Risky-Choice Framing}

Tversky \& Kahneman risky-choice framing (row 2 of Table 1) required subjects to choose between a certain outcome (riskless) and an uncertain outcome (risky) with positive and negative framing. Figure $7 \mathrm{~b}$ summarises the results, which reflect the pattern observed in Tversky and Kahneman's original study (Figure 2).

Under a positive frame a larger proportion of subjects chose the riskless alternative $(56.6 \%)$ than the risky one $(43.4 \%)$. However, there is a lack of confidence in this difference, which does not provide support for $H_{2}: \chi^{2}(1)=1.07, p=.3$. In contrast, under a negative frame $\left(H_{3}\right)$ the difference is larger, reversed, and more confident, with only $29.9 \%$ of subjects choosing the certain loss of the negative frame in preference to the risky loss $(70.1 \%): \chi^{2}(1)=11.69, p=.0006$. Finally, a two-sample test for equality of proportions between positive and negative frames shows support for choice reversal $\left(\mathrm{H}_{4}\right.$ : $\left.\chi^{2}(1)=10.1, p=.002\right)$.

\section{UI-Style Attribute Framing}

Under UI-style framing, subjects chose to either turn 'Snapping On' or 'Snapping Off' in response to a single framed statement (rows 3 and 4 of Table 1). There were three attributeframing conditions (row 3): (3a) a baseline condition that emphasised both positive and negative attributes of the snapping behaviour; ( $3 \mathrm{~b}$ ) a positive condition that emphasised only the alignment of targets with the grid; and (3c) a negative condition that emphasised only misalignment. As before, all three frames are logically the same.

Note that unlike the Tversky \& Kahneman-style analyses (Figure 7), which sum to $100 \%$ as they show the decision between the two opposing frames, the UI-style analyses (Figure 8) show the percentage of subjects that chose to enable snapping in response to a single framing statement.

Under positive framing, $39.7 \%$ of subjects chose to enable snapping, compared to $19.4 \%$ in the negative framing condition (Figure 8a), supporting $H_{1}: \chi^{2}(1)=6.4, p=.01$. Furthermore, as might be expected, the proportion choosing to enable snapping in the baseline condition (27.6\%) was between the proportions for positive and negative framing. 


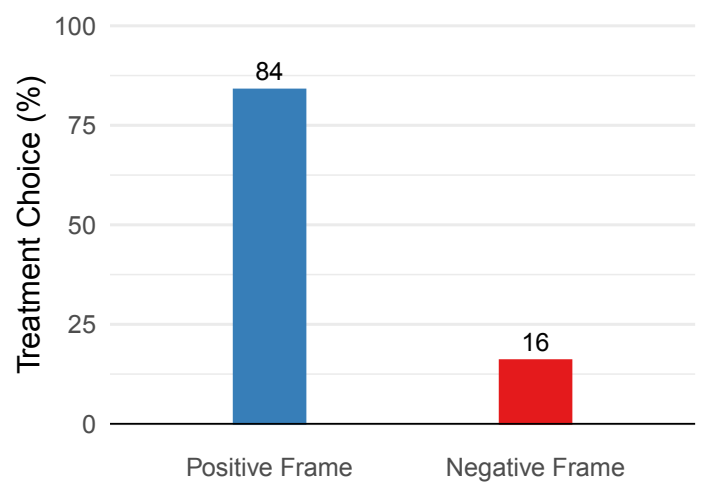

(a) Attribute framing (row 1 in Table 1).

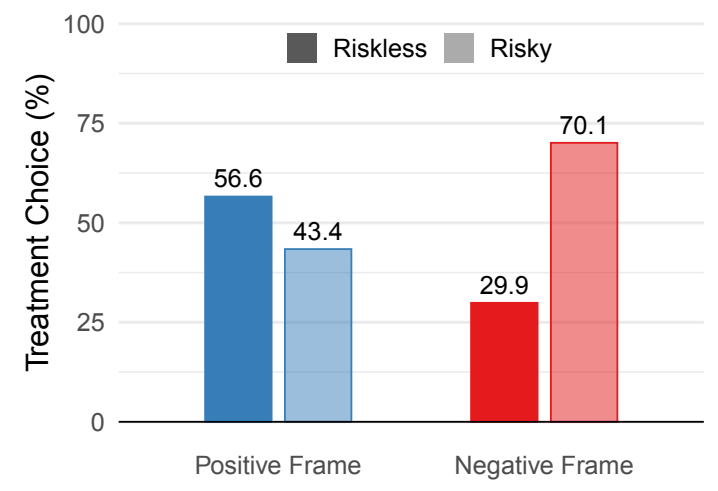

(b) Risky-choice framing (row 2 in Table 1).

Figure 7. Tversky \& Kahneman framing conditions: the percentage of subjects choosing the opposing framing options.

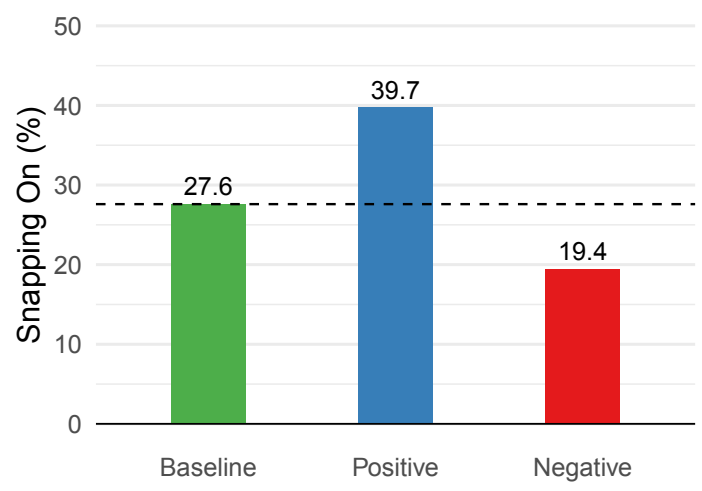

(a) Attribute framing (row 3 in Table 1).

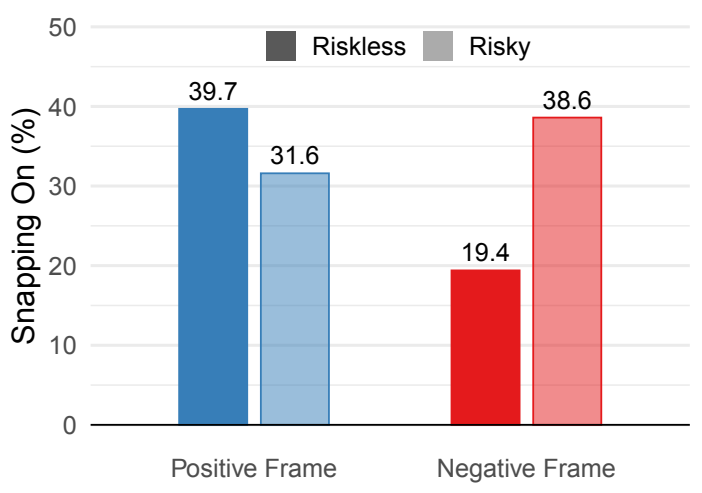

(b) Risky-choice framing (row 4 Table 1).

Figure 8. UI-style framing: percentage of users choosing 'Snapping On' in each condition.

\section{UI-Style Risky-Choice Framing}

The UI-style risky-choice analysis combines data from the riskless-positive and riskless-negative conditions (3b and $3 \mathrm{c}$ of Table 1 - analysed above) with data from the riskypositive and risky-negative frames (rows $4 \mathrm{a}$ and $4 \mathrm{~b}$ ). The intention is to gain insight into how outcome certainty and risk influence users' decisions when framed positively and negatively - but in an interaction setting where choices are to enable or disable features. Figure $8 \mathrm{~b}$ summarises the results, with choice patterns again conforming to those found by Tversky and Kahneman (Figure 2).

Under a positive frame, a larger proportion of subjects chose to enable snapping with a riskless outcome (39.7\%) than with a risky outcome (31.6\%) - risk aversion for gains. Although the proportions align with hypothesis $\mathrm{H}_{2}$, a two-sample test of equality of proportions does not provide support for the hypothesis: $\chi^{2}(1)=0.79, p=.37$. However, responses did support the hypothesis of risk seeking for losses $\left(\mathrm{H}_{3}\right)$ : under a negative frame, a lower proportion of subjects chose to enable snapping with a riskless outcome (19.4\%) than with a risky outcome $(38.6 \%)-\chi^{2}(1)=5.86, p=.015$.

Finally, conforming with the hypothesis of choice reversal $\left(H_{4}\right)$, a binomial GLM of the interaction between frame $\epsilon$
$\{$ positive, negative $\}$ and risk $\in\{$ riskless, risky\} indicates that subjects' acceptance of riskless vs. risky outcomes differed across positive and negative frames: $\chi^{2}(1)=6.9, p=.008$.

\section{Order Effects and Regret}

Although not directly connected with our hypotheses we were curious whether order effects influenced subjects' decisions, and whether they were more or less likely to change their decisions having chosen to enable or disable snapping (at step 7 in Figure 5).

There was little evidence that the order in which subjects experienced the neutral and snapping conditions (steps 2 and 3 of in Figure 5) influenced their decision to enable snapping: $28 \%$ of those who experienced snapping first chose to enable it, compared to $34 \%$ of those who experienced snapping second $\left(\chi^{2}(1)=2.37, p=.12\right)$.

After their decision at step 4 of the procedure (Figure 5), subjects subsequently completed 12 tasks using their decision, and then made a final decision regarding what their choice would be if repeating those tasks one more time. Only $8 \%$ of subjects who chose to disable snapping at step 4 of the procedure opted to change their decision (and enable snapping) at step 7. However, a much larger proportion (34\%) of those 
who chose to enable snapping at step 4 opted to change their decision (and disable snapping) to step 7. A two-sample test of these proportions shows $\chi^{2}(1)=55.3, p<1 \times 10^{-5}$.

The low proportion reversing their decision to disable snapping at step 7 is unsurprising: as the grid is invisible and there is no difference to neutral task performance, subjects have little reason to believe that snapping may have been beneficial for the final set of 12 tasks. However, when they chose to enable snapping at step 4 of the procedure in the risky-choice conditions (rows $4 \mathrm{a}$ and $4 \mathrm{~b}$ of Table 1), there was a $66 \%$ chance that all of the final 12 tasks would be misaligned and a $33 \%$ chance that all would be aligned. Only $13 \%$ of sbjects who chose to enable snapping and subsequently experienced 12 aligned tasks opted to reverse their decision at step 7. Conversely, $87 \%$ of those who enabled snapping and subsequently experienced 12 misaligned tasks opted to reverse their decision.

\section{DISCUSSION}

Our results suggest that attribute framing $\left(H_{1}\right)$ with both Tversky \& Kahnemen and UI-style frames influenced subjects' decisions regarding interface features. Regarding risk aversion for gains $\left(\mathrm{H}_{2}\right)$, the results for neither framing type provided support for the hypothesis. However, the results did support the hypothesis of risk seeking for losses $\left(H_{3}\right)$. Responses also indicated support for the hypothesis of choice-reversal under the influence of risk for positive and negative frames in both framing types $\left(H_{4}\right)$.

\section{Preliminary Studies}

Prior to the present experiment we conducted two preliminary studies: one in a lab $(n=100)$ and one on Amazon Mechanical Turk $(n=360)$. As with this experiment, the preliminary studies were based on snap-to-grid functionality and used a similar procedure (Figure 5). However, the preliminary studies only examined UI-style framing (rows 3 and 4 in Table 1), and the Mechanical Turk study used videos demonstrating the interactive behaviours (rather than actual interaction).

The results of these preliminary studies were useful for assessing the magnitude of the difference between subjects' decisions (for power analyses), and also showed that they were much more likely to accept snapping after passive observation of interaction (the videos in the Mechanical Turk study) than they were after real interaction (the lab study). Results from the preliminary studies showed lower levels of statistical confidence than that observed in the present experiment due to limitations in their methodology and statistical power. Full descriptions of these studies are available in the auxiliary materials to this paper.

\section{Choice Proportions Across Framing Types}

Tversky and Kahneman's original results (Figure 2) suggest that the tendency for risk seeking in negative frames may be stronger than the tendency for risk aversion in positive frames: the gap between riskless and risky negative frames is larger than that for positive frames. Our results similarly carry this suggestion (Figures $7 \mathrm{~b}$ and $8 \mathrm{~b}$ ), which aligns with extensive prior work in psychology showing that negative outcomes tend to elicit strong effects than positive ones [1]. However, as it was not a planned manipulation, further work is required to analyse the reliability of such an effect.

\section{Implications for Design}

Prior to the current study, it was unclear that UI-style framing would induce any of the hypothesised effects. Unlike the many previous psychological studies, UI-style framing cues referred to relatively weak stimuli (aligned vs. misaligned targets, rather than life vs. death [31]). Furthermore, our subjects' decisions concerned enabling/disabling an interface feature in response to a single framing statement, rather than a forced choice between two opposing framing statements.

However, the results show that even with these weak stimuli and without direct-choice opposition forced by the method, relatively subtle changes in UI-style framing resulted in substantial differences in the proportion of subjects opting to enable the snapping interface feature. Designers should therefore carefully consider the labelling associated with interface choices, particularly if they believe that one option is more desirable for their users.

This lends general support to previous analyses of framing in the fields of recommender systems $[2,4]$ and persuasive design $[6,30]$, and might be broadly applicable in interaction. For instance, when new features are added to a system, designers may be able to influence the likelihood that users will enable them (or, not disable them) by adding a few words to the associated labels that emphasise the feature's positive attributes. Conversely, if interface features are likely to be removed in upcoming releases, users could be encouraged to disable them in advance by altering their labels to emphasise their negative attributes. Feedback from user telemetry on interaction history might be used to inform these labels with frames appropriate for a user's task history [e.g. 22].

Although users may sometimes be certain that an interface feature will assist (or hinder) their upcoming tasks, it is more common that users will lack certainty and will be faced with risky choices. In general, users may not know what their future tasks will require or may be unfamiliar with all aspects of the feature (particularly if it is new), and therefore the likelihood that the system's features will be beneficial to those tasks is uncertain (user risk). Similarly, the system itself may contain elements of non-determinism that make it difficult to estimate the probability of an assistive response (system risk). For example, a keyboard that offers a word prediction feature requires users to make judgements about the text they will enter in the future (user risk) and the system's ability to produce correct predictions (system risk). Each of these factors means that users may be unsure about whether the interface will assist or hinder in upcoming tasks, creating an element of risk in their choice, and therefore influencing the effectiveness of different forms of framing.

Designers must consider whether and when it is appropriate to employ framing effects. Should designers be attempting to manipulate users into adopting or abandoning features, or should interfaces strive to present neutral frames that emphasise neither gains nor losses? These are important issues, but the merits, role, and desirability of framing (intentional or 
otherwise) will be context dependent. For example, there is a growing range of applications that seek to assist users in making better lifestyle choices, including quitting undesirable habits such as smoking [25], managing addictions and psychological disorders, and maintaining medication regimes [29]. Better understanding of framing effects could help designers produce interfaces that are more effective in assisting users to attain their goals. For example, the SwiftKey mobile keyboard (Figure 3) provides information to users describing how much more productive they are with SwiftKey (rather than how much less productive they would be without it), how many typos it has corrected, and how many words it has predicted. As interfaces are already presenting overt frames for user decisions, it seems important to better understand the effects of their alternative forms.

Finally, our experiments represent only a single set of findings from a narrow set of experimental conditions, and there is extensive scope for replication - including method generalisation. First, our experiments used drag-and-drop tasks with and without snap-to-grid functionality to provide an easy to control means for examining user choices. Further studies with other interface features, such as text entry auto-correct, are needed to examine the generality of our findings. Second, the framing statements used in our study were closely based on those used by Tversky and Kahneman, and future studies should examine the effects of moving to the types of framing statements that are more likely to occur during interaction. Third, we intentionally used two radio buttons for the user's choice, labelled 'Snapping On' and 'Snapping Off' (in that order). Further work is needed to examine how even these labels influence decisions, including their interaction with the framing that precedes them. While it may seem adventurous to propose that such subtle features can influence decisions, results from prior research suggest that they might. In particular, research into acquiescence bias has shown that survey respondents are more likely to select options with positive connotations [23], and Shafir [28] showed that subjects weighted information concerning negative aspects more strongly when asked to reject items (and similarly for positive aspects when asked to choose).

\section{CONCLUSION}

Framing effects, in which positive or negative attributes of potential outcomes are emphasised, are known to reliably influence people's decisions. Furthermore, peoples' decisions concerning the risk associated with outcomes are known to differ when positive and negative attributes are highlighted: people tend to be risk averse when positive frames emphasise potential gains, but risk seeking when negative frames emphasise losses. We analysed how these effects influence users' decisions to enable or disable interface features when positive or negative attributes are emphasised, and when upcoming tasks are certain or uncertain.

Experimental results conformed with hypotheses. Positive attribute framing resulted in much higher acceptance of interface options than negative framing, despite logically equivalent outcomes. Subjects' decisions also conformed with risk seeking for losses, consistent with preferring the risk of a potentially larger loss to the certainty of a smaller loss. And their acceptance of risk showed a pattern consistent with choice-reversal across positive and negative frames.

\section{REFERENCES}

[1] Roy F. Baumeister, Ellen Bratslavsky, Catrin Finkenauer, and Kathleen D. Vohs. 2001. Bad is stronger than good. Review of General Psychology 5, 4 (2001), 323-370. DOI : https://doi.org/10.1037/1089-2680.5.4. 323

[2] André Calero Valdez, Martina Ziefle, and Katrien Verbert. 2016. HCI for Recommender Systems: The Past, the Present and the Future. In Proceedings of the 10th ACM Conference on Recommender Systems (RecSys '16). ACM, New York, NY, 123-126. DOI : https://doi.org/10.1145/2959100.2959158

[3] Eun Kyoung Choe, Jaeyeon Jung, Bongshin Lee, and Kristie Fisher. 2013. Nudging People Away from Privacy-Invasive Mobile Apps through Visual Framing. In Human-Computer Interaction - INTERACT 2013 (Lecture Notes in Computer Science), Paula Kotzé, Gary Marsden, Gitte Lindgaard, Janet Wesson, and Marco Winckler (Eds.), Vol. 8119. Springer, Berlin/Heidelberg, Germany, 74-91. DOI:https : //doi.org/10.1007/978-3-642-40477-1_5

[4] Dan Cosley, Shyong K. Lam, Istvan Albert, Joseph A. Konstan, and John Riedl. 2003. Is Seeing Believing?: How Recommender System Interfaces Affect Users' Opinions. In Proceedings of the SIGCHI Conference on Human Factors in Computing Systems (CHI '03). ACM, New York, NY, 585-592. DOI :https : //doi.org/10.1145/642611.642713

[5] Antonella De Angeli, Alistair Sutcliffe, and Jan Hartmann. 2006. Interaction, Usability and Aesthetics: What Influences Users' Preferences?. In Proceedings of the 6th Conference on Designing Interactive Systems (DIS '06). ACM, New York, NY, 271-280. DOI: https://doi.org/10.1145/1142405.1142446

[6] Juho Hamari, Jonna Koivisto, and Tuomas Pakkanen. 2014. Do Persuasive Technologies Persuade? - A Review of Empirical Studies. In Persuasive Technology, Anna Spagnolli, Luca Chittaro, and Luciano Gamberini (Eds.). Springer International Publishing, Cham, Germany, 118-136. DOI:https://doi.org/10. 1007/978-3-319-07127-5_11

[7] Richard J. Harris. 1973. Answering questions containing marked and unmarked adjectives and adverbs. Journal of Experimental Psychology 97, 3 (1973), 399-401. DOI:https://doi.org/10.1037/h0034165

[8] Jan Hartmann, Antonella De Angeli, and Alistair Sutcliffe. 2008. Framing the User Experience: Information Biases on Website Quality Judgement. In Proceedings of the SIGCHI Conference on Human Factors in Computing Systems (CHI '08). ACM, New York, NY, 855-864. DOI:https://doi.org/10/ fktzv4

[9] Jan Hartmann, Alistair Sutcliffe, and Antonella De Angeli. 2007. Investigating Attractiveness in Web User Interfaces. In Proceedings of the SIGCHI 
Conference on Human Factors in Computing Systems (CHI '07). ACM, New York, NY, 387-396. DOI : https://doi.org/10.1145/1240624.1240687

[10] Jan Hartmann, Alistair Sutcliffe, and Antonella De Angeli. 2008. Towards a Theory of User Judgment of Aesthetics and User Interface Quality. ACM Transactions on Computer-Human Interaction 15, 4, Article 15 (2008), 30 pages. DOI:https://doi.org/10/b57cf9

[11] Maria L. Hwang and Lena Mamykina. 2017. Monster Appetite: Effects of Subversive Framing on Nutritional Choices in a Digital Game Environment. In Proceedings of the 2017 CHI Conference on Human Factors in Computing Systems (CHI'17). ACM, New York, NY, 4082-4096. DOI : https://doi.org/10.1145/ 3025453.3026052

[12] Anthony Jameson, Bettina Berendt, Silvia Gabrielli, Federica Cena, Cristina Gena, Fabiana Vernero, and Katharina Reinecke. 2014. Choice Architecture for Human-Computer Interaction. Foundations and Trends in Human-Computer Interaction 7, 1-2 (2014), 1-235. DOI :https://doi.org/10.1561/1100000028

[13] Daniel Kahneman and Amos Tversky. 1984. Choices, Values, and Frames. American Psychologist 39, 4 (1984), 341-350. DOI:https://doi.org/10.1037/ 0003-066X.39.4.341

[14] Gideon B. Keren and Jeroen G. W. Raaijmakers. 1988. On between-subjects versus within-subjects comparisons in testing utility theory. Organizational Behavior and Human Decision Processes 41, 2 (1988), 233-247. DOI:https://doi.org/10/b2nkrd

[15] Young-Ho Kim, Jae Ho Jeon, Eun Kyoung Choe, Bongshin Lee, KwonHyun Kim, and Jinwook Seo. 2016. TimeAware: Leveraging Framing Effects to Enhance Personal Productivity. In Proceedings of the SIGCHI Conference on Human Factors in Computing Systems (CHI '16). ACM, New York, NY. DOI: https://doi.org/10.1145/2858036.2858428

[16] Irwin P. Levin. 1987. Associative effects of information framing. Bulletin of the Psychonomic Society 25, 2 (1987), 85-86. DOI:https://doi.org/10.3758/ BF03330291

[17] Irwin P. Levin and Gary J. Gaeth. 1988. How Consumers Are Affected by the Framing of Attribute Information Before and After Consuming the Product. Journal of Consumer Research 15, 3 (1988), 374-378. DOI : https://doi.org/10.1086/209174

[18] Irwin P. Levin, Sandra L. Schneider, and Gary J. Gaeth. 1998. All Frames Are Not Created Equal: A Typology and Critical Analysis of Framing Effects. Organizational Behavior and Human Decision Processes 76, 2 (1998), 149-188. DOI:https://doi.org/10/bzntst

[19] Irwin P. Levin, Sara K. Schnittjer, and Shannon L. Thee. 1988. Information framing effects in social and personal decisions. Journal of Experimental Social Psychology 24, 6 (1988), 520-529. DOI : https : //doi.org/10.1016/0022-1031(88)90050-9

[20] Elizabeth F. Loftus. 1975. Leading questions and the eyewitness report. Cognitive Psychology 7, 4
(1975), 560-572. DOI:https://doi.org/10.1016/ 0010-0285(75) 90023-7

[21] Theresa M. Marteau. 1989. Framing of information: Its influence upon decisions of doctors and patients. British Journal of Social Psychology 28, 1 (1989), 89-94. DOI : https://doi.org/10.1111/j.2044-8309.1989. tb00849.x

[22] Justin Matejka, Wei Li, Tovi Grossman, and George Fitzmaurice. 2009. CommunityCommands: Command Recommendations for Software Applications. In Proceedings of the 22nd Annual ACM Symposium on User Interface Software and Technology (UIST '09). ACM, New York, NY, 193-202. DOI :https : //doi.org/10.1145/1622176.1622214

[23] Samuel Messick and Douglas N. Jackson. 1961. Acquiescence and the factorial interpretation of the MMPI. Psychological Bulletin 58, 4 (1961), 299-304. DOI :https://doi.org/10.1037/h0043979

[24] Tobias Nyström and Moyen M. Mustaquim. 2015. Managing Framing Effects in Persuasive Design for Sustainability. In Proceedings of the 19th International Academic Mindtrek Conference (AcademicMindTrek '15). ACM, New York, NY, 122-129. DOI : https : //doi.org/10.1145/2818187.2818277

[25] Jeni Paay, Jesper Kjeldskov, Mikael B. Skov, Lars Lichon, and Stephan Rasmussen. 2015. Understanding Individual Differences for Tailored Smoking Cessation Apps. In Proceedings of the 33rd Annual ACM Conference on Human Factors in Computing Systems (CHI '15). ACM, New York, NY, 1699-1708. DOI : https://doi.org/10.1145/2702123.2702321

[26] Philip Quinn and Andy Cockburn. 2016. When Bad Feels Good: Assistance Failures and Interface Preferences. In Proceedings of the 2016 CHI Conference on Human Factors in Computing Systems (CHI '16). ACM, New York, NY, 4005-4010. DOI:https: //doi.org/10/bhqq

[27] Eeva Raita and Antti Oulasvirta. 2011. Too good to be bad: Favorable product expectations boost subjective usability ratings. Interacting with Computers 23,4 (2011), 363-371. DOI:https://doi.org/10.1016/ j.intcom. 2011.04 .002

[28] Eldar Shafir. 1993. Choosing versus rejecting: Why some options are both better and worse than others. Memory \& Cognition 21, 4 (1993), 546-556. DOI: https://doi.org/10.3758/BF03197186

[29] Katarzyna Stawarz, Anna L. Cox, and Ann Blandford. 2014. Don't Forget Your Pill!: Designing Effective Medication Reminder Apps That Support Users' Daily Routines. In Proceedings of the SIGCHI Conference on Human Factors in Computing Systems (CHI '14). ACM, New York, NY, 2269-2278. DOI : https://doi.org/10.1145/2556288.2557079

[30] Kristian Torning and Harri Oinas-Kukkonen. 2009. Persuasive System Design: State of the Art and Future Directions. In Proceedings of the 4th International Conference on Persuasive Technology (Persuasive '09). ACM, New York, NY, Article 30, 8 pages. DOI : 
https://doi.org/10.1145/1541948.1541989

[31] Amos Tversky and Daniel Kahneman. 1981. The framing of decisions and the psychology of choice. Science 211, 4481 (1981), 453-458. DOI :https: //doi.org/10/fj3z3r

[32] Amos Tversky and Daniel Kahneman. 1986. Rational
Choice and the Framing of Decisions. The Journal of Business 59, 4, Pt. 2 (1986), S251-S278.

[33] Ronald L. Wasserstein, Allen L. Schirm, and Nicole A. Lazar. 2019. Moving to a World Beyond " $p<0.05$ ". The American Statistician 73 (2019), 1-19. DOI : https : //doi.org/10.1080/00031305.2019.1583913 